\title{
Interacting agegraphic dark energy models in phase space
}

\section{O. A. Lemets D. A. Yerokhin L. G. Zazunov}

Akhiezer Institute for Theoretical Physics, National Science Center "Kharkov Institute of Physics and Technology", Akademicheskaya Str. 1, 61108 Kharkov, Ukraine

E-mail: oleg.lemets@gmail.com, denyerokhin@gmail.com

\begin{abstract}
Agegraphic dark energy, has been recently proposed, based on the so-called Karolyhazy uncertainty relation, which arises from quantum mechanics together with general relativity. In the first part of the article we study the original agegraphic dark energy model by including the interaction between agegraphic dark energy and pressureless (dark) matter. The phase space analysis was made and the critical points were found, one of which is the attractor corresponding to an accelerated expanding Universe.

Recent observations of near supernova show that the acceleration of Universe decreases. This phenomenon is called the transient acceleration. In the second part of Article we consider the 3-component Universe composed of a scalar field, interacting with the dark matter on the agegraphic dark energy background. We show that the transient acceleration appears in frame of such a model. The obtained results agree with the observations.
\end{abstract}

Keywords: dark energy, agegraphic, transient acceleration,scalar field, interaction

ArXiv ePrint: 1010.0567 


\section{Contents}

1 Introduction $\quad 1$

2 Review on the interacting ADE $\quad 3$

2.1 A phase-space view on the interacting ADE 3

2.2 Phase plane analysis 5

3 The model of interacting dark energy with a transient acceleration phase 7

3.1 Review of the case $Q=0 \quad 9$

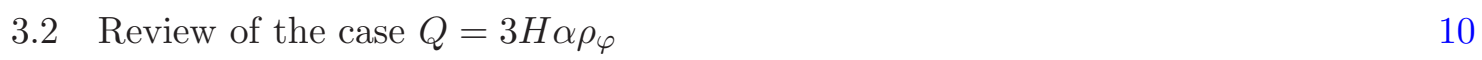

4 Observational data $\quad 10$

5 Conclusion 11

\section{Introduction}

Nowadays it is strongly believed that the Universe is experiencing an accelerated expansion, and this opinion is supported by many cosmological observations such as SNe Ia [1], WMAP [3], SDSS [5] and X-ray [7]. These observations suggest that the Universe is dominated by dark energy with negative pressure, which provides the dynamical mechanism for the accelerating expansion for the Universe.

The simplest candidate of dark energy is a tiny positive cosmological constant. However, as is well known, it suffers from the so-called cosmological constant [8] and coincidence problem [9]. The cosmological constant problem is essentially a quantum gravity problem, since the cosmological constant is commonly considered as the vacuum expectation value of some quantum fields. Since a completely successful quantum theory of gravity is still unavailable, it is more realistic directly to combine quantum mechanics with general relativity.

Although the nature and origin of dark energy could perhaps understood by a fundamental underlying theory unknown up to now, physicists can still propose some paradigms to describe it. In this direction we can consider theories of modified gravity [11, 12], or field models of dark energy. The field models that have been discussed widely in the literature consider a cosmological constant [13], a canonical scalar field (quintessence) [15], a phantom field, that is a scalar field with a negative sign of the kinetic term [17, 19].

However, we still can make some attempts to probe the nature of dark energy according to some principles of quantum gravity although a complete theory of quantum gravity is not available today. The holographic dark energy model is just an appropriate and interesting example, which is constructed in the light of the holographic principle of quantum gravity theory [20-22]. Before a completely successful quantum theory of gravity is available, it is more realistic to combine quantum mechanics with general relativity directly.

Following the line of quantum fluctuations of spacetime, in Refs. [24-26, 35], we use the so-called Károlyházy relation [23]

$$
\delta t=\beta t_{p}^{2 / 3} t^{1 / 3}
$$


where $\beta$ is a numerical factor of order one, $t_{p}$ is the reduced Planck time, and throughout this paper, we use the units $c=\hbar=k_{b}=1$, so that one has $l_{p}=t_{p}=1 / M_{p}$ with $l_{p}$ and $M_{p}$ being the reduced Planck length and mass, respectively.

The Károlyházy relation (1.1) together with the time-energy uncertainty relation enables one to estimate a quantum energy density of the metric fluctuations of Minkowski spacetime $[25,26,35]$. With the relation (1.1), a length scale $t$ can be known with a maximal precision $\delta t$ determining a minimal detectable cell $\delta^{3}$ over a spatial region $t^{3}$. Thus one is able to look at the microstructure of space-time over a region $t^{3}$ by viewing the region as the one consisting of cells $\delta t^{3} \sim t_{p}^{2} t$. Therefore such a cell $\delta t^{3}$ is the minimal detectable unit of space-time over a given length scale $t$ and if the age of the space-time is $t$, its existence due to the time-energy uncertainty relation cannot be justified with energy smaller than $\sim t^{-1}$ . Hence, as a result of the relation (1.1), one can conclude that if the age of the Minkowski space-time is $t$ over a spatial region with linear size $t$ (determining the maximal observable patch) there exists a minimal cell $\delta t^{3}$, the energy of the cell cannot be smaller than $[24,26,35]$

$$
E_{\delta t^{3}} \sim t^{-1},
$$

due to the time-energy uncertainty relation, it was argued that the energy density of metric fluctuations of Minkowski spacetime is given by

$$
\rho_{q} \sim \frac{E_{\delta t^{3}}}{\delta t^{3}} \sim \frac{1}{t_{p}^{2} t^{2}} \sim \frac{M_{p}^{2}}{t^{2}} .
$$

In [26] (see also [35]), it is noticed that the Károlyházy relation naturally obeys the holographic black hole entropy bound [29]. It is worth noting that the form of energy density eq. (1.3) is similar to the one of holographic dark energy [32,34], i.e., $\rho_{\Lambda} \sim l_{p}^{-2} l^{-2}$. The similarity between $\rho_{q}$ and $\rho_{\Lambda}$ might reveal some universal features of quantum gravity, although they arise from different sources. As the most natural choice, the time scale $t$ in eq. (1.3) is chosen to be the age of our Universe. Therefore, we call it "agegraphic" dark energy [35].

There are many variations of models with agegraphic dark energy (ADE) to solve most problems of SCM. Particularly, various articles consider the possibility of interaction of this kind of dark energy with dark matter $[37,39]$.

Some authors have recently suggested that the cosmic acceleration have already peaked and that we are currently observing its slowing down [40-42]. Under a kinematic analysis of the most recent SNe Ia compilations, in the paper [42] shows the existence of a considerable probability in the relevant parameter space that the Universe is already in a decelerating expansion regime.

We does not know works, which investigated the possibility to explain this phenomenon in the framework of ADE model. Thus, it is interesting to consider the model with the interacting dark energy, for transient acceleration. Typically, in models with transient acceleration the role of dark energy played by scalar field, non-monotonic dynamics of which provide the necessary dependence of deceleration parameter. In our work we made an attempt to combine the scalar field model with agegraphic dark energy.

The paper is organized as follows: in the next section we consider the dynamic features of the standard agegraphic dark energy interacting with the substance with an arbitrary equation of state $w$. In section 3 we investigate a new quintessence scenario driven by a rolling homogeneous scalar field in the exponential potential interacting with dark matter on the agegraphic background. It will be shown that this scenario predict transient accelerating phase. Consistency of these models with the observational data will be shown in Secs. 4 . 


\section{Review on the interacting ADE}

In this Section we consider the dynamics of the three component Universe, which consists of ADE, dark matter and radiation. Since the nature of dark energy is not fully known, we can assume that the dark energy and dark matter interact. The energy density of ADE is given by [35]

$$
\rho_{q}=\frac{3 n^{2} M_{p}^{2}}{T^{2}} .
$$

Here $M_{p}=(8 \pi G)^{-1 / 2}$ and $T$ is chosen to be the age of our Universe

$$
T=\int_{0}^{t} d t^{\prime}=\int_{0}^{a} \frac{d a}{H a},
$$

where $a$ is the scale factor of our Universe, $H \equiv \dot{a} / a$ is the Hubble parameter and the dot denotes the derivative with respect to cosmic time.

Considering the flat Friedmann-Robertson-Walker Universe with the agegraphic dark energy and substance with an arbitrary equation of state $w$ and energy density $\rho_{w}$, the corresponding Friedmann equation is

$$
H^{2}=\frac{1}{3 M_{p}^{2}}\left(\rho_{q}+\rho_{w}+\rho_{\gamma}\right)
$$

The conservation laws of the ADE and matter are respectively [37]

$$
\begin{aligned}
\dot{\rho}_{w}+3 H(1+w) \rho_{w} & =Q, \\
\dot{\rho}_{q}+3 H\left(1+w_{q}\right) \rho_{q} & =-Q, \\
\dot{\rho}_{\gamma}+4 H \rho_{\gamma} & =0,
\end{aligned}
$$

where $Q$ denotes the phenomenological interaction term. Note that the interaction is introduced in the form that satisfies covariance of the total energy momentum tensor. In the literature most often one can find the fallowing forms of interaction [37, 38, 43-45, 54, 55]

$$
Q=3 \alpha H \rho_{q}, \quad 3 \beta H \rho_{w}, \quad 3 \gamma H\left(\rho_{w}+\rho_{q}\right),
$$

where $\alpha, \beta$ and $\gamma$ are positive constant parameters. We consider the most general of above cited types of interaction

$$
Q=3 H\left(\alpha \rho_{q}+\beta \rho_{w}\right) .
$$

\subsection{A phase-space view on the interacting ADE}

In this section, we investigate the global structure of the dynamical system via phase plane analysis and compute the cosmological evolution by numerical analysis. We introduce the following variables:

$$
x=\frac{1}{M_{p} H} \sqrt{\frac{\rho_{q}}{3}}, \quad y=\frac{1}{M_{p} H} \sqrt{\frac{\rho_{w}}{3}}, \quad z=\frac{1}{M_{p} H} \sqrt{\frac{\rho_{\gamma}}{3}} .
$$

Taking the derivative of both sides of the Friedman equation (2.3) with respect to the logarithm of the scale factor, $N=\ln a$, and using eqs. (2.4), it is easy to find that

$$
\frac{d H}{d N}=-\frac{3 H}{2}\left(\left(1+w_{q}\right) x^{2}+(1+w) y^{2}+\frac{4}{3} z^{2}\right) .
$$


Using (2.8) and (2.9) the governing equations of motion (2.4), (2.1) could be reexpressed as the following system of equations:

$$
\begin{aligned}
x^{\prime} & =\frac{3 x}{2} f(x, y, z)-\frac{x^{2}}{n}, \\
y^{\prime} & =\frac{3 y}{2} f(x, y, z)-\frac{3}{2}(1+w) y, \\
z^{\prime} & =\frac{3 z}{2} f(x, y, z)-2 z,
\end{aligned}
$$

where

$$
f(x, y, z)=\frac{2}{3 n} x^{3}-\alpha x^{2}+(1+w-\beta) y^{2}+\frac{4}{3} z^{2}
$$

and the prime denotes a derivative with respect to the logarithm of the scale factor, $N=\ln a$. The first Friedmann equation can be written as

$$
x^{2}+y^{2}+z^{2}=1 .
$$

Since it is limited by the condition $\rho>0$ to the circle $x^{2}+y^{2}+z^{2} \leq 1$, we limit the analysis only to the quarter of unitary sphere of positive $x, y, z$.

The state parameter $w_{q}$ for the agegraphic dark energy could be expressed in terms of the new variables as

$$
w_{q}=-1-\alpha+\frac{2}{3 n} x-\beta \frac{y^{2}}{x^{2}} .
$$

The total equation of state parameter is given by

$$
w_{t o t}=\frac{p_{t o t}}{\rho_{t o t}}=\frac{p_{q}+p_{w}+p_{\gamma}}{\rho_{q}+\rho_{w}+\rho_{\gamma}}=\frac{w_{q} x^{2}+w y^{2}+\frac{1}{3} z^{2}}{x^{2}+y^{2}+z^{2}} .
$$

For completeness, we give the deceleration parameter

$$
q=-\frac{\ddot{a}}{a H^{2}}=-1-\frac{\dot{H}}{H^{2}}
$$

Combining it with the Hubble parameter and noting that $\dot{H}=H^{\prime} H$ we obtain the deceleration parameter in terms of the (2.8) variables

$$
q=-1+\frac{3}{2}\left[\frac{2}{3 n} x^{3}+\alpha x^{2}+(1+w-\beta) y^{2}+\frac{4}{3} z^{2}\right]
$$

It is worth noting that in the absence of interaction between agegraphic dark energy and dark matter, $\alpha=\beta=0$, from eq. (2.12) we see that $w_{q}$ is always greater than -1 . In addition the condition $n>1$ is necessary to derive the present accelerated expansion. However, the situation change as soon as the interaction term is taken into account.

In interacting agegraphic models of dark energy, the properties of agegraphic dark energy are determined by the parameters $n, \alpha$ and $\beta$ together. 


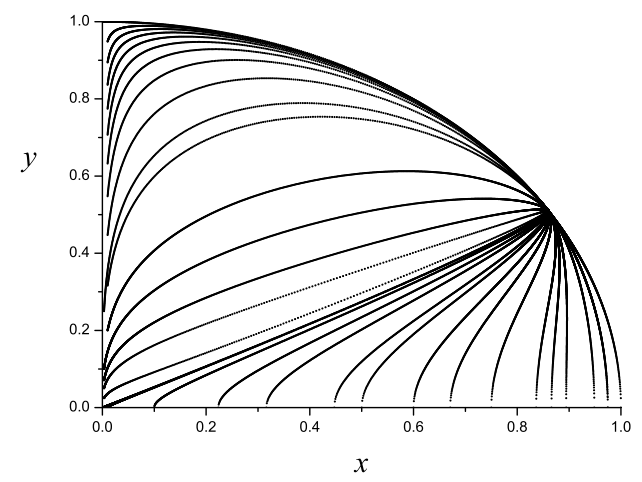

Figure 1. The phase plane for $n=3, \alpha=0.25$ and $\beta=0.1$. The late-time attractor is the scaling solution with $x_{*} \approx 0.86, y_{*} \approx 0.26\left(\Omega_{q *} \approx 0.74, \Omega_{m *} \approx 0.26\right)$.

Table 1. Location of the critical points of the autonomous system of eqs. (3.4), their stability and dynamical behavior of the Universe at those points.

\begin{tabular}{cccc}
\hline $\begin{array}{c}\left(x_{c}, y_{c}, z_{c}\right) \\
\text { coordinates }\end{array}$ & $\begin{array}{c}\text { Stability } \\
\text { character }\end{array}$ & $q$ & $w_{q}$ \\
\hline$(0,0,1)$ & unstable & 1 & $-1-\alpha$ \\
$(0,1,0)$ & unstable & $-1+\frac{3}{2}(1+w-\beta)$ & $\nexists$ \\
$\left(x_{*}, y_{*}, 0\right)$ & attractor & $q_{*}<0$ & $w_{q *}$ \\
\hline
\end{tabular}

\subsection{Phase plane analysis}

As said above, the motivation for interacting agegraphic models is to solve or, at least, ameliorate the coincidence problem. The goal will be achieved if the system (2.10) presents scaling solutions. As already discussed, scaling solutions are characterized by a constant dark matter to dark energy ratio $r=y^{2} / x^{2}$. It is easy to find that

$$
r^{\prime}=3 \alpha-3\left(1+w-\beta-\frac{2}{3 n} x\right) r
$$

The scaling solutions mean $r^{\prime}=0$, as a result $r_{*}=\alpha\left(1+w-\beta-\frac{2}{3 n} x_{*}\right)^{-1}$. On the other hand in this mode $x^{\prime}=y^{\prime}=0, z \approx 0$ and we obtain from the first equation in (2.10) that

$$
x_{*}=\frac{1}{2}\left(-b+\sqrt{b^{2}+4}\right),
$$

where $b=\frac{3 n}{2}\left((1+w-\beta) r_{*}-\alpha\right)$. For the case shown in figure 1, we find $r_{*} \approx 0.353$ and accordingly $x_{*} \approx 0.86$.

Even more important are those scaling solutions that are also an attractor. In this way, the coincidence problem gets substantially alleviated because, regardless of the initial conditions, the system evolves toward a final state where the ratio of dark matter to dark energy stays constant (see figure 1). The location of attractor, as shown above (2.17), depends on the values $\alpha$ and $\beta$. The simplest finite critical points and their properties for this model are summarized in Table 1. 

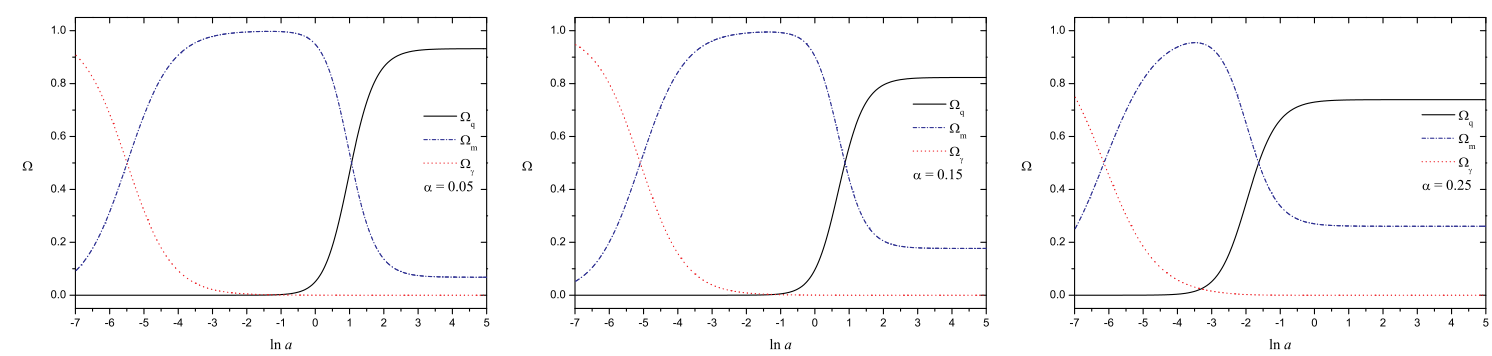

Figure 2. Behavior of $\Omega_{\gamma}$ (dot line), $\Omega_{m}$ (dash dot line) and $\Omega_{q}$ (solid line) as a function of $N=\ln a$ for $n=3, \beta=0.1$ and $\alpha$ as indicated.

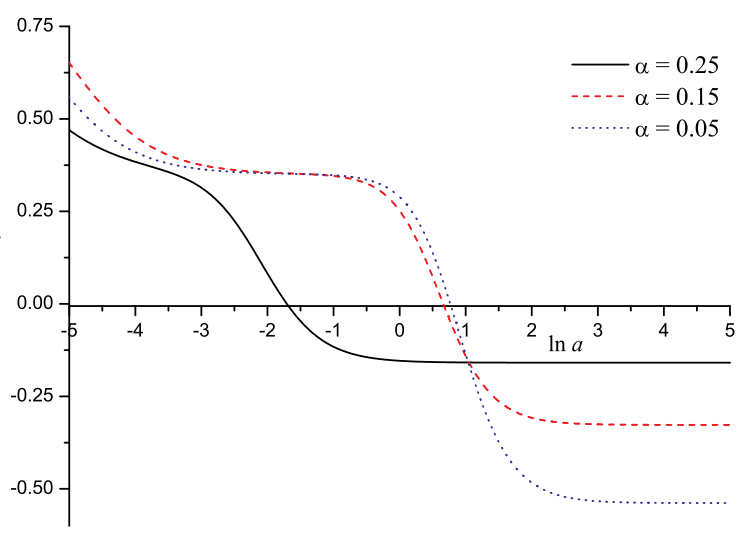

Figure 3. Behavior of deceleration parameter $q$ as a function of $\ln a$ for $\beta=0.1$ and $\alpha$ as indicated.

The first critical point, $(0,0,1)$, is unstable and corresponds to the radiation dominated era. The other point, $(0,1,0)$, is physically unrealistic. It corresponds to a Universe filled with matter with parameter of state $w$ (it contains neither radiation, nor dark energy) and it is also unstable. Finally, the third critical point, $\left(x_{*}, y_{*}, 0\right)$, is stable and in a natural way, the dynamical evolution of the Universe asymptotically approaches this attractor, a never-ending phase of accelerated expansion, in which the energy ratio $r=y^{2} / x^{2}$ remains constant (for $\alpha \neq 0$ ). To conclude, the succession of expansion eras in the model under consideration can be summarized as follows: if the initial conditions satisfy $z_{0}>x_{0} \neq 0$, then after the initial radiation dominated era, the Universe might have gone through a very short period of matter domination with state parameter $w$ (if any) corresponding to an unstable critical point, to finally asymptotically approach a regime with a constant dark matter to dark energy ratio, at late times, in the accelerated expansion era. Note that if initially the Universe does not contain dark energy then in the further evolution of dark energy does not exist. This fact is the fundamental difference from the models, in which the role of dark energy is played by scalar field [50].

In figure 2 , we show the evolution of $\Omega_{q}, \Omega_{m}$ and $\Omega_{\gamma}$ for different model parameters $\alpha$ in the case of $w=0$ and $\beta=0.1$. It is easy to see that for the fixed $\beta$, which describes the decay dark matter to agegraphic dark energy, increasing the value of the parameter $\alpha$ leads to increase in the contribution of the relative density of dark matter $\Omega_{m}$.

In figure 3 we show the evolution of the deceleration parameter $q$ as a function of $N=\ln a$ for $\beta=0.1$ and $\alpha$ as indicated. The key distinction of this model is that the 
accelerated expansion of the Universe at late times is inherent even in the case when the phase of dark energy dominance does not occur.

\section{The model of interacting dark energy with a transient acceleration phase}

Recent investigations seem to favor the cosmological dynamics according to which the accelerated expansion of the Universe may have already peaked and is now slowing down again [41]. As a consequence, the cosmic acceleration may be a transient phenomenon. One of the first on the possibility of a transient acceleration drew attention J. D. Barrow [40]. In his work he showed that in many well-motivated scenarios the observed spell of vacuum domination is only a transient phenomenon. Soon after acceleration starts, the vacuum energy's antigravitational properties are reversed, and a matter-dominated decelerating cosmic expansion resumes. This was achieved by considering the dark energy in form of scalar quintessence field $\varphi$ defined by its self-interaction potential $V(\varphi)$. The potential of the scalar field in this case is the product of exponential and quadratic potentials.

The possible cosmological roles of exponential potentials have been investigated before, but almost always as a means of driving a period of cosmological inflation [51, 52]. This requires potentials that are much flatter than those usually found in particle physics models.

In this section, we follow the latter route and investigate a new quintessence scenario driven by a rolling homogeneous scalar field with exponential potential $V(\varphi)$ interacting with dark matter on the agegraphic background. This scenario predicts transient accelerating phase.

To describe the dynamic properties of such a Universe will adapt the system of equations (3.4) for this model. We introduce the modified variables:

$$
x=\frac{\dot{\varphi}}{\sqrt{6} M_{p} H}, \quad y=\frac{1}{M_{p} H} \sqrt{\frac{V(\varphi)}{3}}, \quad z=\frac{1}{M_{p} H} \sqrt{\frac{\rho_{m}}{3}}, \quad u=\frac{1}{M_{p} H} \sqrt{\frac{\rho_{q}}{3}} .
$$

The evolution of the scalar field is described by the Klein-Gordon equation, which in the presence of matter couplings is given by

$$
\ddot{\varphi}+3 H \dot{\varphi}+\frac{d V}{d \varphi}=-\frac{Q}{\dot{\varphi}} .
$$

In this section, we consider interactions $Q$ that are linear combinations of the scalar field and pressureless matter:

$$
Q=3 H\left(\alpha \rho_{\varphi}+\beta \rho_{m}\right),
$$

where $\alpha, \beta$ are constant parameters.

Without specifying the potential of the scalar field $V(\varphi)$, we obtain the system of equations in the form

$$
\begin{aligned}
x^{\prime} & =\frac{3 x}{2} g(x, z, u)-3 x+\sqrt{\frac{3}{2}} \lambda y^{2}-\gamma, \\
y^{\prime} & =\frac{3 y}{2} g(x, z, u)-\sqrt{\frac{3}{2}} \lambda x y, \\
z^{\prime} & =\frac{3 z}{2} g(x, z, u)-\frac{3}{2} z+\gamma \frac{x}{z}, \\
u^{\prime} & =\frac{3 u}{2} g(x, z, u)-\frac{u^{2}}{n}
\end{aligned}
$$


where $g(x, z, u)=2 x^{2}+z^{2}+\frac{2}{3 n} u^{3}$ and

$$
\gamma \equiv-\frac{Q}{\sqrt{6} M_{p} H^{2} \dot{\varphi}}, \lambda \equiv-\frac{1}{V} \frac{d V}{d \varphi} M_{p} .
$$

In these variables, we obtain

$$
\begin{aligned}
& Q=9 H^{3} M_{p}^{2}\left[\alpha\left(x^{2}+y^{2}\right)+\beta z^{3}\right], \\
& \gamma=\frac{\alpha\left(x^{2}+y^{2}\right)+\beta z^{3}}{x} .
\end{aligned}
$$

We will consider a scalar field with an exponential potential energy density

$$
V=V_{0} \exp \left(\sqrt{\frac{2}{3}} \frac{\mu \varphi}{M_{p}}\right),
$$

where $\mu$ is a constant. In this case we obtain

$$
\begin{aligned}
x^{\prime} & =\frac{3 x}{2}\left[g(x, z, u)-\frac{\alpha\left(x^{2}+y^{2}\right)+\beta z^{2}}{x^{2}}\right]-3 x-\mu y^{2}, \\
y^{\prime} & =\frac{3 y}{2} g(x, z, u)+\mu x y, \\
z^{\prime} & =\frac{3 z}{2}\left[g(x, z, u)+\frac{\alpha\left(x^{2}+y^{2}\right)+\beta z^{2}}{z^{2}}\right]-\frac{3}{2} z, \\
u^{\prime} & =\frac{3 u}{2} g(x, z, u)-\frac{u^{2}}{n} .
\end{aligned}
$$

In this model the deceleration parameter has the form

$$
q=-1+\frac{3}{2}\left[2 x^{2}+z^{2}+\frac{2}{3 n} u^{3}\right] .
$$

Note that in this model, the cosmological parameters are not explicitly depend of the parameters of interaction, but only determine by the behaviors of dynamical variables. This fact complicates the analysis of our model.

The fact that $q$ is independent of the interaction term implies that the region of phase space is the same for all of the models considered. It is possible to make some qualitative comments about the system (3.8) for some special cases.

Initially, considering the case, $y=0$, we obtain the constraining relation, which should be imposed on the parameters of interaction for the emergence of critical points. It is easy to show that in this case, the condition of real energy density requires

$$
2 \sqrt{\frac{\beta}{\alpha}}>1+\alpha+\beta
$$

which necessarily requires $0>\beta>\alpha,|\alpha|+|\beta|<1$. This critical point corresponds to the matter-dominated Universe and is unstable. The cases of several such points are possible, but they are of no interest. Finally, it can be shown that any of this equilibrium point within (but not on) the boundary will exist for $x_{0}<0$. For $z \neq 0$ and constrain satisfied (3.10), it

$$
x_{c}=\left[(a+\sqrt{\beta / \alpha})^{1 / 2}+a\right] z_{c},
$$

where $a=(2 \sqrt{\beta / \alpha}-(1+\alpha+\beta))^{1 / 4}$. 
Table 2. Location of the critical points of the autonomous system of Eqs. (3.8), their stability and dynamical behavior of the Universe at those points.

\begin{tabular}{ccccc}
\hline $\begin{array}{c}\left(x_{c}, y_{c}, z_{c}, u_{c}\right) \\
\text { coordinates }\end{array}$ & $\begin{array}{c}\text { Stability } \\
\text { character }\end{array}$ & $q$ & $w_{\varphi}$ & $w_{\text {tot }}$ \\
\hline$(1,0,0,0)$ & unstable & 2 & 1 & 1 \\
$(0,1,0,0)$ & unstable & -1 & -1 & -1 \\
$(0,0,1,0)$ & unstable & $\frac{1}{2}$ & $\nexists$ & 0 \\
$(0,0,0,1)$ & stable & $-1+\frac{1}{n}$ & $\nexists$ & $-1+\frac{2}{3 n}$ \\
$\left(-\frac{3}{2 \mu}, \frac{3}{2 \mu}, \sqrt{1-\frac{3}{2 \mu^{2}}}, 0\right)$ & unstable & $\frac{1}{2}$ & $\nexists$ & 0 \\
$\left(x_{*}, y_{*}, 0, u_{*}\right)$ & attractor & $q_{*}<0$ & $w_{\varphi *}$ & $w_{\text {tot* }}$ \\
\hline
\end{tabular}

\subsection{Review of the case $Q=0$}

In this subsection we consider in more detail the case of absence of interaction between the scalar field and dark matter. Critical points of the system (3.8) in this $(\alpha=\beta=0)$ case are given in the Table 2. As can be seen from the table, the system (3.8), there are six physically admissible critical points, the latter of which is the attractor. The first critical point, $(1,0,0,0)$, is unstable and corresponds to the scalar field dominated era with extremely rigid equation of state, the second critical point corresponds to the period of evolution when the scalar field behaves as a cosmological constant. The other point, $(0,0,1,0)$, is physically unrealistic. It corresponds to a Universe filled with dark matter (it contains neither scalar field, nor agegraphic dark energy) and it is also unstable. Fourth critical point $(0,0,0,1)$ correspond to the Universe consisting only of the agegraphic dark energy and has already been discussed in detail.

The physical interest present the final sixth critical point, which is the an attractor. It corresponds to the Universe consisting of a scalar field and agegraphic dark energy. The location of this critical point is completely determined by the parameter of the potential $\mu$ and the value of $n$ :

$$
\begin{array}{ll}
x_{*}=\frac{2}{3 n \mu} u_{*}, & y_{*}=\sqrt{1-\left(1+\frac{4}{9 n^{2} \mu^{2}}\right) u_{*}^{2}}, \\
z_{*}=0, & u_{*}=\frac{3}{2 n \mu^{2}}\left(-1+\sqrt{1+\frac{4 n^{2} \mu^{4}}{9}}\right) .
\end{array}
$$

The fact that $x_{*} \propto u_{*}$ is the characteristic feature of the tracking solutions. Note also that between the scalar field and agegraphic dark energy, evidence of background interactions. There is because the dynamics of the scalar field affects agegraphic dark energy. This effect is that agegraphic dark energy, having a negative pressure, affect on the rate of expansion of the Universe, which affects the Hubble parameter, which is included in the Klein-Gordon equation for a scalar field.

The attractor, once reached, brings to zero the matter density. To allow for the observed matter content of the Universe, we have to select the initial conditions, if they exist, in such a way that the attractor is not yet reached at the present time, but the expansion is already accelerated.

For example, consider the case when $\mu=-3, n=3$. The dynamics of such a Universe is consistent with the observed data, which will be shown in the following section. We note only 

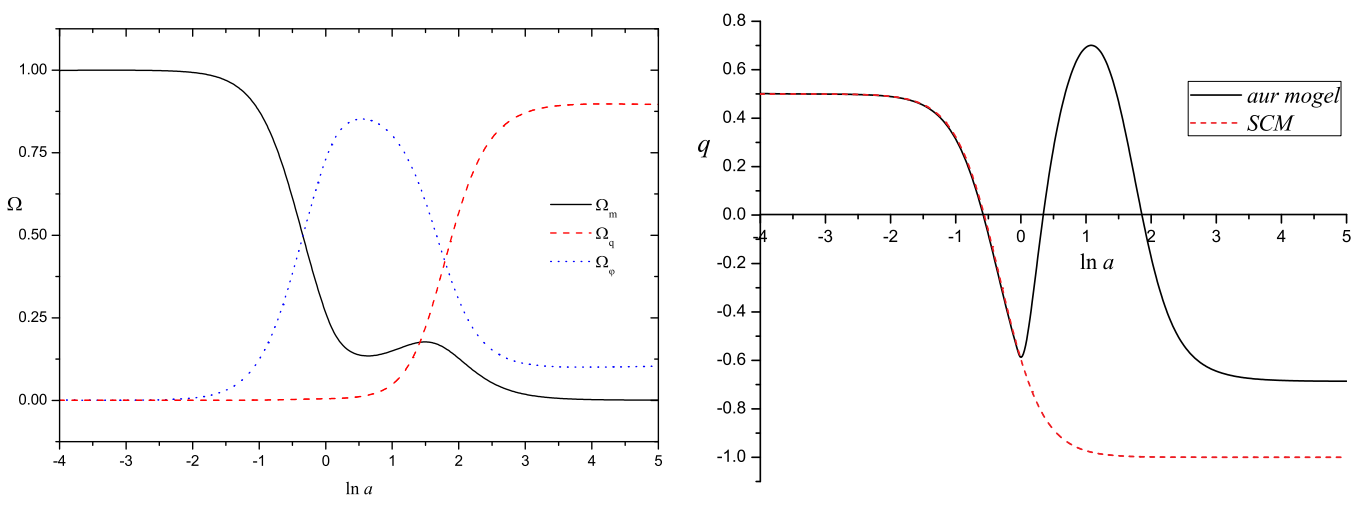

Figure 4. Behavior of $\Omega_{\varphi}$ (dot line), $\Omega_{q}$ (dash line) and $\Omega_{m}$ (solid line) as a function of $N=\ln a$ for $n=3, \alpha=0.005$ and $\mu=-3$ (left side). Evolution of deceleration parameter for this model (right side).

that in this model the values of deceleration parameter and the scalar field state parameter in the attractor are equal respectively $q_{*} \approx-0.68, w_{\varphi *} \approx-0.78$.

\subsection{Review of the case $Q=3 H \alpha \rho_{\varphi}$}

In the above case, the phenomenon of transient acceleration that occurs in such a Universe does not match the observations. However, it is possible to fit the observational data with this model in the case when dark matter and scalar field interact. In this subsection we consider the special case of interaction (3.3) when $\beta=0$.

In figure 4 , we show the evolution of $\Omega_{q}, \Omega_{m}$ and $\Omega_{\varphi}$ for cosmological model in the case when $\alpha=0.005, \mu=-3$ and $n=3$.

From the form of equations and the character of the interaction it can be easily understood that neither the nature nor the location of the attractor, that have been found in the previous subsection dos not change with the inclusion of the interaction. Interaction only affects the behavior of dynamical variables that are the correspond to trajectory in phase space between critical points. This is a consequence of the above degeneracy from the parameters of interaction.

With these values of the parameters of interaction, transient acceleration begins almost in the present era. In our model as in most cosmological models, where the scalar field plays the role of dark energy it begins to dominate causing a period of accelerated expansion of the Universe. Its consequence of the accelerated expansion of the Universe contribution of ADE increases, resulting that the background (space) is changing faster than the field and it becomes asymptotically free. This field has extremely rigid equation of state that leads to the fact that the accelerated expansion of the Universe is slowing down. Soon, however, when the contribution of $\mathrm{ADE}$ has grows enough so that the scalar field cannot longer impede the expansion of the Universe, it begins to accelerate again.

\section{Observational data}

In the present section, we will consider the latest cosmological observations, namely, the 397 Constitution SNIa dataset [56]. 

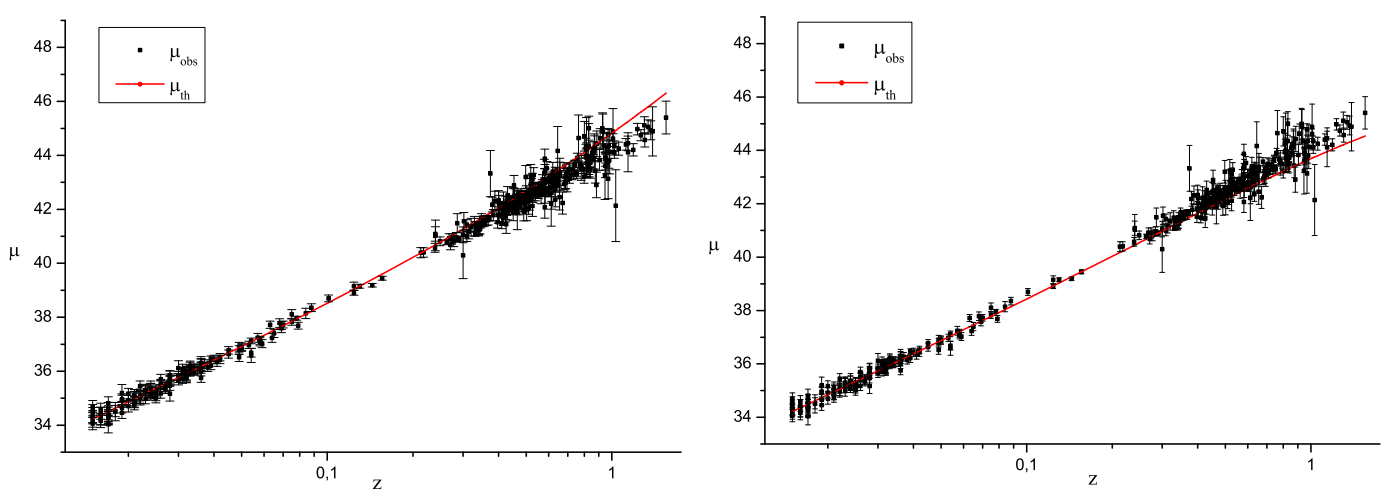

Figure 5. The dependence of the modulus distance from the redshift, theoretically calculated (solid red line) by the model with $Q=3 H\left(\alpha \rho_{q}+\beta \rho_{m}\right), \alpha=0.25, n=3$ (left side) and $Q=3 H \alpha \rho_{\varphi}$, $\alpha=0.005, \mu=-3$ and $n=3$ (right side). $h=0.65 \mathrm{~km} \mathrm{c}^{-1} \mathrm{Mpc}^{-1}$ for both models and found the results of observations of supernovae type 1a [56]

The data points of the 397 Constitution SNIa compiled in [56] are given in terms of the distance modulus $\mu_{o b s}\left(z_{i}\right)$. On the other hand, the theoretical distance modulus is defined as

$$
\mu_{t h}\left(z_{i}\right) \equiv 5 \log _{10} D_{L}\left(z_{i}\right)+\mu_{0},
$$

where $\mu_{0} \equiv 42.38-5 \log _{10} h$ and $h$ is the Hubble constant $H_{0}$ in units of $100 \mathrm{~km} \mathrm{~s}^{-1} \mathrm{Mpc}^{-1}$, whereas

$$
D_{L}(z)=(1+z) \int_{0}^{z} \frac{d \tilde{z}}{E(\tilde{z} ; \mathbf{p})}
$$

in which $E \equiv H / H_{0}$, and $\mathbf{p}$ denotes the model parameters.

Theoretical distance modulus will be different for the different models and comparing $\mu_{t h}\left(z_{i}\right)$ with $\mu_{o b s}\left(z_{i}\right)$, one can judge the plausibility of an cosmological model. So to understand whether the theoretical model corresponds to the observational data is enough to know the value of $E \equiv H / H_{0}$, which is easy to find through a system of equations (3.8). As seen from figure 5 our models are in accordance with the observational data.

\section{Conclusion}

The original agegraphic dark energy model was proposed in [35] based on the Károlyházy uncertainty relation, which arises from quantum mechanics together with general relativity. The interacting agegraphic dark energy model has certain advantages compared to the original agegraphic or holographic dark energy model. Many studies show that this model gives an opportunity to explain the accelerated expansion of the Universe without a cosmological constant or some form of the scalar field. All the three models give dynamics of the Universe which are virtually indistinguishable from SCM, but without most of its problems, such as the cosmological constant, fine tuning and coincidence problems.

Some authors have recently suggested that the cosmic acceleration have already peaked and that we are currently observing its slowing down [40-42]. Under a kinematic analysis of the most recent SNe Ia compilations, the paper [42] shows the existence of a considerable probability in the relevant parameter space that the Universe is already in a decelerating expansion regime. 
One of the deficiencies of original ADE model is the inability to explain the phenomenon of transient acceleration.

Density of holographic dark energy is determined by the surface terms in action, while volume terms are usually ignored. We take into account both surface and volume terms, where the latter correspond to (described by) homogeneous scalar field with exponential potential $V(\varphi)$.

We consider a model of Universe consisting of dark matter interacting with a scalar field on the agegraphic background. It is shown that this model can explain the transient acceleration. This model also is in accordance with the observational data.

\section{Acknowledgements}

We are grateful to our research supervisor Prof. Yu.L. Bolotin for kind help and discussions. We also thank V.A. Cherkaskiy for careful reading and editing of this article.

\section{References}

[1] A. G. Riess et al. Observational evidence from supernovae for an accelerating Universe and a cosmological constant, Astron. J. 116 (1998) 1009 [astro-ph/9805201].

[2] S. Perlmutter et al., Measurements of omega and lambda from 42 high-redshift supernovae, Astrophys. J. 517 (1999) 565 [astro-ph/9812133].

[3] D. N. Spergel et al., First year Wilkinson microwave anisotropy probe (WMAP) observations: determination of cosmological parameters, Astrophys. J. Suppl. 148 (2003) 175 [astro-ph/0302209].

[4] D. N. Spergel et al., Wilkinson microwave anisotropy probe (WMAP) three year results: implications for cosmology, Astrophys. J. Suppl. 170 (2007) 377 [astro-ph/0603449].

[5] M. Tegmark et al., Cosmological parameters from SDSS and WMAP, Phys. Rev. D 69 (2004) 103501 [astro-ph/0310723].

[6] K. Abazajian et al., The second data release of the Sloan Digital Sky Survey, Astron. J. 128 (2004) 502 [astro-ph/0403325].

[7] S. W. Allen, et al., Cosmological constraints from the local X-ray luminosity function of the most X-ray luminous galaxy clusters, Mon. Not. Roy. Astron. Soc. 353 (2004) 457 [astro-ph/0208394v3].

[8] S. Weinberg, The cosmological constant problem, Rev. Mod. Phys. 61 (1989) 1.

[9] P. Steinhardt, in Critical Problems in Physics edited by V.L.Fitch and D.R.Marlow, Princeton University Press, Princeton, NJ, (1997), pg. 301.

[10] I. Zlatev, L. Wang and P. J. Steinhardt, Quintessence, cosmic coincidence, and the cosmological constant, Phys. Rev. Lett. 82 (1999) 896 [astro-ph/9807002v2].

[11] P.G. Bergmann, Comments on the scalar-tensor theory, Int. J. Theor. Phys. 1 (1968) 25.

[12] S.Nojiri, S. D. Odintsov, Introduction to modified gravity and gravitational alternative for dark energy, Int. J. Geom. Meth. Mod. Phys. 4 (2007) 115 [arXiv:hep-th/0601213].

[13] P. J. Peebles and B. Ratra, The cosmological constant and dark energy, Rev. Mod. Phys. 75 (2003) 559 [astro-ph/0207347v2].

[14] J. Kratochvil, A. Linde, E. V. Linder and M. Shmakova, Testing the cosmological constant as a candidate for dark energy, JCAP 0407 (2004) 001 [astro-ph/0312183] 
[15] R. R. Caldwell, R. Dave and P. J. Steinhardt, Cosmological imprint of an energy component with general equation of state, Phys. Rev. Lett. 80 (1998) 1582 [astro-ph/9708069v2].

[16] M. S. Turner and M. White, CDM models with a smooth component, Phys. Rev. D56 (1997) 4439 [astro-ph/9701138v2].

[17] R. R. Caldwell, Measuring the speed of sound of quintessence, Phys. Lett. B 545 (2002) 23 [astro-ph/0112438v1].

[18] S. Nojiri and S.D. Odintsov, Quantum deSitter cosmology and phantom matter, Phys. Lett. B562 (2003) 147 [arXiv:hep-th/0303117].

[19] R.R. Caldwell, M. Kamionkowski and N.N. Weinberg, Phantom Energy and Cosmic Doomsday, Phys. Rev. Lett. 91 (2003) 071301 [astro-ph/0302506].

[20] L. Susskind, The World as a Hologram, J. Math. Phys. 36 (1995) 6377 [hep-th/9409089v2].

[21] G. 't Hooft, Dimensional reduction in quantum gravity [gr-qc/9310026].

[22] E. Witten, Anti de Sitter space and holography, Adv. Theor. Math. Phys. 2 (1998) 253 [hep-th/9802150v2].

[23] F. Károlyházy, Gravitation and Quantum Mechanics of Macroscopic Objects, Nuovo Cimento, A 42 (1966) 390.

[24] Naoki Sasakura, An uncertainty relation of space-time, Prog. Theor. Phys. 102 (1999) 169 [hep-th/9903146].

[25] M. Maziashvili, Space-time in light of Karolyhazy uncertainty relation, Int. J. Mod. Phys. D 16 (2007) 1531 [gr-qc/0612110].

[26] M. Maziashvili, Cosmological implications of Karolyhazy uncertainty relation, Phys. Lett. B 652 (2007) 165 [arXiv:0705.0924].

[27] V. Sahni and A. A. Starobinsky, The case for a positive cosmological lambda-term Int. J. Mod. Phys. D 9 (2000) 373 [astro-ph/9904398].

[28] P. J. E. Peebles and B. Ratra, The Cosmological Constant and Dark Energy, Rev. Mod. Phys. 7 (2003) 559 [astro-ph/0207347].

[29] Bekenstein, J.D., Black holes and entropy, Phys. Rev. D 7 (1973) 2333.

[30] M.Li, A model of holographic dark energy, Phys. Lett. B 603 (2004) 1 [hep-th/0403127].

[31] A.G. Cohen, D.B. Kaplan and A.E. Nelson, Effective field theory, black holes, and the cosmological constant, Phys. Rev. Lett. 82 (1999) 4971 [hep-th/9803132].

[32] H. Wei and S.N. Zhang, Age problem in the holographic dark energy model, Phys. Rev. D 76 (2007) 063003 [arXiv:0707.2129].

[33] S. D. H. Hsu, Entropy Bounds and Dark Energy, Phys. Lett. B 594 (2004) 13 [hep-th/0403052].

[34] X. Zhang and F.Q. Wu, Constraints on holographic dark energy from the latest supernovae, galaxy clustering, and cosmic microwave background anisotropy observations, Phys. Rev. D 76 (2007) 023502 [astro-ph/0701405].

[35] R. G. Cai, A dark energy model characterized by the age of the universe, Phys. Lett. B 657 (2007) 228 [arXiv:0707.4049].

[36] H. Wei and R. G. Cai, A new model of agegraphic dark energy, Phys. Lett. B 660 (2008) 113 [arXiv:0708.0884].

[37] H. Wei and R. G. Cai, Interacting Agegraphic Dark Energy, Eur. Phys. J. C 59 (2009) 99 [arXiv:0707.4052].

[38] Luis P. Chimento, Linear and nonlinear interactions in the dark sector, Phys. Rev. D 81 
(2010) 043525 [arXiv:0911.5687].

[39] A. Sheykhi, Interacting agegraphic dark energy models in non-flat Universe, Phys. Lett. B680 (2009) 113.

[40] J. Barrow, R. Bean and J. Magueijo, Can the Universe escape eternal acceleration?, Mon. Not. Roy. Astron. Soc. 316 (2000) 41 [astro-ph/0004321].

[41] Arman Shafieloo, Varun Sahni, Alexei A. Starobinsky, Is cosmic acceleration slowing down?, Phys. Rev. D 80, 101301 (2009), [arXiv:0903.5141]

[42] Antonio C. C. Guimara es, Jose' Ademir S. Lima, Could the cosmic acceleration be transient? A cosmographic evaluation, [arXiv:1005.2986].

[43] L. Amendola, Scaling solutions in general non-minimal coupling theories, Phys. Rev. D 60, (1999) 043501 [astro-ph/9904120].

[44] L. Amendola, Coupled quintessence, Phys. Rev. D 62 (2000) 043511 [astro-ph/9908023].

[45] W. Zimdahl and D. Pavon, Interacting quintessence, Phys. Lett. B 521 (2001) 133 [astro-ph/0105479].

[46] W. Zimdahl and D. Pavon, Scaling Cosmology, Gen. Rel. Grav. 35 (2003) 413 [astro-ph/0210484].

[47] L. P. Chimento, A. S. Jakubi, D. Pavon and W. Zimdahl, Interacting quintessence solution to the coincidence problem, Phys. Rev. D 67 (2003) 083513 [astro-ph/0303145].

[48] B. Gumjudpai, T. Naskar, M. Sami and S. Tsujikawa, Coupled dark energy: Towards a general description of the dynamics, JCAP 06 (2005) 007 [hep-th/0502191].

[49] L. Amendola, M. Quartin, S. Tsujikawa and I. Waga, Challenges for scaling cosmologies, Phys. Rev. D 74 (2006) 023525 [astro-ph/0605488].

[50] C.G. Boehmer, G. Caldera-Cabral, R. Lazkoz, R. Maartens, Dynamics of dark energy with a coupling to dark matter, Phys. Rev. D 78 (2008) 023505 [arXiv:0801.1565v2].

[51] F. Lucchin and S. Matarrese, Power-law inflation, Phys. Rev. D 32 (1985) 1316.

[52] J.J. Halliwell, Scalar fields in cosmology with an exponential potential, Phys. Lett. B 185 (1987) 341.

[53] A. B. Burd and J. D. Barrow, Inflationary models with exponential potentials, Nucl. Phys. B 308 (1988) 929.

[54] H. Wei and R. G. Cai, K-Chameleon and the Coincidence Problem, Phys. Rev. D 71 (2005) 043504 [hep-th/0412045].

[55] Z. K. Guo, R. G. Cai and Y. Z. Zhang, Cosmological evolution of interacting phantom energy with dark matter, JCAP 05 (2005) 002 [astro-ph/0412624].

[56] M. Hicken et al., Improved dark energy constraints from 100 new CfA supernova type Ia light curves, Astrophys. J. 700 (2009) 1097 [arXiv:0901.4804]. 